\title{
The ionized gas in the spiral galaxy NGC 3359
}

\section{Kinematics}

\author{
M. $\operatorname{Rozas}^{1}$, A. Zurita ${ }^{1}$, J.E. Beckman ${ }^{1}$, and D. Pérez ${ }^{2}$ \\ 1 Instituto de Astrofísica de Canarias, E-38200 La Laguna, Tenerife, Canarias, Spain \\ 2 Department of Physical Sciences, University of Hertfordshire, Hatfield, Herts, AL10 9AB, UK
}

Received June 7; accepted December 2, 1999

\begin{abstract}
The spiral galaxy NGC 3359 has been observed in the $\mathrm{H} \alpha$ line with the TAURUS II Fabry-Perot interferometer at the William Herschel Telescope within the BARS programme on the Canary Island telescopes in order to study the kinematics of the ionized gas.

We have produced maps of velocity and velocity dispersion, and computed the rotation curve out to $\sim 3$ scale lengths from the centre of the galaxy. From the map of the residual velocities, obtained after subtracting off the systemic rotation, we detected streaming motions in the gas across the spiral arms and the presence of non-circular motions of order $30-40 \mathrm{~km} \mathrm{~s}^{-1}$ around the bar: the gas response to the gravitational potential. The velocity dispersion map shows typical values of order $15 \mathrm{~km} \mathrm{~s}^{-1}$ for the HII regions, but also reveals major wings of high dispersion associated with the most luminous, density-limited regions.
\end{abstract}

Key words: galaxies: individual (NGC 3359) — galaxies: spiral — interstellar medium: HıI regions

\section{Introduction}

Hydrogen is by far the most abundant component of the interstellar medium (ISM). Studying the structural and kinematic properties of each of its phases helps us understand the physical phenomena which give rise to those phases.

The study of the ISM, its kinematics, and the role it plays in star formation enables us to infer the relation between the optical appearance of a galaxy and the evolution of its spiral or barred structure, in response to the underlying dynamics of its stellar and gaseous components.

Send offprint requests to: J.E. Beckman
One alternative to the usual kinematic technique for spirals, which uses the Hr $21 \mathrm{~cm}$ line, is Fabry-Perot interferometry of $\mathrm{H} \alpha$, which permits the acquisition of much data in a short observing time, useful above all for kinematic mapping of a whole galaxy. With this method one can obtain standard information, such as intensity and velocity maps, as well as rotation curves with high spatial and velocity resolution. From this one can go on to analyze the role of ionized hydrogen as a tracer of the star forming process, via its general distribution, and also, using the kinematics, obtain information on specific processes in defined zones of star formation, such as arms and bars. Fabry-Perot interferometry is valuable for studying motion perpendicular to the plane of the galaxy observed, as well as non-circular motion in the plane. These can be streaming motions across the arms or gas flows around the bar, whose analysis yields the underlying dynamics of the galaxy, via the response of the ionized hydrogen to the non-axisymmetric component of its gravitational potential (Knapen 1997, 1998). One can compute maps of residual velocity, after subtracting the projected circular component, and from these estimate the non-circular motions on a global scale. The observations also yield maps of velocity dispersion, needed to understand the vertical equilibrium, and departures from equilibrium in the galaxy, and the kinematics of specific His regions (Combes \& Bequaert 1997; Rozas et al. 1998a). Finally non-circular motions, specifically vertical motions above star formation zones are important to show how the disc is affecting the halo, and to compare with theoretical models (e.g. Norman \& Ikeuchi 1988). Therefore the study of the internal kinematic of stars and gas in spiral galaxies continues being an area of active investigation in astrophysics.

This work provides in this field new detailed observations which report the internal gas motions in the galaxy NGC 3359, a barred late type spiral (SBc) galaxy, included in the most relevant grand design galaxy surveys. The complex morphology of this galaxy shows significant 
features at different wavelengths, such as an apparently inclined nuclear bar misaligned in the $I$ band (Sempere \& Rozas 2000) or twin peaks in the central part of the $\mathrm{H} \alpha$ distribution of the galaxy, which are out of alignment with the principal bar, underlining the absence of star formation in the nucleus. In this work the kinematic data of this galaxy, useful by itself, are provided. Moreover, a complete analysis of the gas distribution and kinematics in the optical disc is performed for this galaxy.

This article is the second half of a study we have performed on the grand-design spiral galaxy NGC 3359, based on high quality images ( $\mathrm{H} \alpha$ and complementary $U, I, K$ images in Rozas et al. 1999; hereafter Paper I) obtained in the context of the BARS international time project of the Canary Island Observatories. We summarize the general properties of the galaxy in Paper I and we show some statistical and physical properties of the HiI regions and analyze the distributions of the ionized gas. In the present study we obtain and analyze a complete Fabry-Perot kinematic map of the ionized hydrogen in NGC 3359. In Sect. 2 we describe the observational and data reduction procedures. Section 3 contains the kinematic results: we analyze the velocity map, derive the rotation curve, and a model velocity field, via which we obtain the residual map of non-circular velocities. We also show the velocity dispersion map in Sect. 3. Section 4 is devoted to the gas flows round the bar, and in Sect. 5 we set our conclusions.

\section{Observations and reduction of the data}

The Fabry-Perot (FP) observations were taken on the night of May 10th 1997, with the $4.2 \mathrm{~m}$ William Herschel Telescope on La Palma. The night was photometric, but with a seeing of $1.3^{\prime \prime}$. The galaxy was observed in $\mathrm{H} \alpha$ using the TAURUS II FP instrument at the Cassegrain focus. The etalon used had a spacing of $125 \mu \mathrm{m}$, and the camera focal ratio was $\mathrm{f} / 2.11$; the detector was a TEK CCD. We windowed the camera to a size of $540 \times 540$ pixels (to avoid vignetting by the filter wheel), at a pixel size of $0.56^{\prime \prime} \times 0.56^{\prime \prime}$, yielding a field size of 5 arcmin square. The observations consisted of exposures with the spacing varied in steps, to scan the full wavelength range of the $\mathrm{H} \alpha$ line as emitted by the whole galaxy. A narrow band filter, centred on the wavelength of $\mathrm{H} \alpha$ appropriately redshifted: $\lambda_{\mathrm{c}}=6589 \AA$ and $\Delta \lambda=15 \AA$, was used for ordersorting. The full spectral range, $17.228 \AA$, was scanned in 55 steps, so that the wavelength interval between consecutive planes was $0.34 \AA$ at the centre of the field. The full effective exposure time was $140 \mathrm{~s}$ per plane. We took a calibration cube with a CuNe lamp at the beginning of the night, and performed the calibration in phase and wavelength using calibration rings taken before and after the data cube. Both types of calibration were carried out using the TAUCAL package in the FIGARO environment, converting the raw data, in which the surfaces of constant wavelength are paraboloids, into a cartesian cube.

The sky background emission was subtracted separately from each plane of the cube, and all the planes were put into common spatial coordinates using field star images. Positional astrometry was performed by comparing the positions of these field stars and of the brightest HII regions in the TAURUS field with their positions on a very well resolved $\mathrm{H} \alpha$ image of the galaxy (Fig. 1 from Paper I), yielding maximum uncertainties in position of $0.5^{\prime \prime}$.

The resulting data cube was used as the input for the program MOMENTS in the GIPSY programme suite, to calculate moment maps. While the channel maps in Fig. 1 give global information about the way the emission changes with velocity across the face of the galaxy, a more detailed description of the spatial distribution of intensities and velocities is provided by the moment maps. The first gives the integrated intensity of the emission at each point, specified in R.A. and dec.; MOMENTS then allows us to calculate the zeroth, first and second order moments at each pixel, which yield the peak intensity of the emission line, the velocity (centre of the emission line) and the dispersion in intensity from the profile respectively. Only signals coming from the same positional point in three adjacent planes were considered true emission, and used to compute the moment maps.

A careful inspection of the moment maps obtained showed that there were zones where the integration over all velocities gave place to quite strong noise features. This made necessary the revision of the initial data cube to eliminate all the noisy areas which were distorted. To do this, we produced cubes at lower spatial resolution, by smoothing the original cube in order to derive intensity and velocity maps at different resolutions, following traditional radio-astronomical practice. In this way, from an initial resolution of $1.5^{\prime \prime}$ we obtained cubes at $6,8,10$ and $16^{\prime \prime}$; for the first two we used an unchanged pixel size, while for the others we binned into $2 \times 2$ pixel bins. Although we did not use all the low resolution data cubes to obtain the results shown in this paper, they were a necessary intermediate step to obtain the final data cube. Then we subtracted the continuum emission from each cube, using those planes in the original cube from which $\mathrm{H} \alpha$ emission was absent. A linear interpolation to these latter gave us the appropriate continuum level to subtract from each cube plane to leave only the $\mathrm{H} \alpha$.

We now briefly describe the procedure followed for the full resolution data set. The first step was to produce a conditionally transferred data cube, in which values were retained only at positions where the intensity in the smoothed data cube at $6^{\prime \prime}$ was larger than 2.5 times the rms noise of the smoothed maps. Pixel values at all other positions were set undefined. Then, noise peaks outside the area where $\mathrm{H} \alpha$ emission is expected were removed by setting pixel values at those positions to undefined too. 


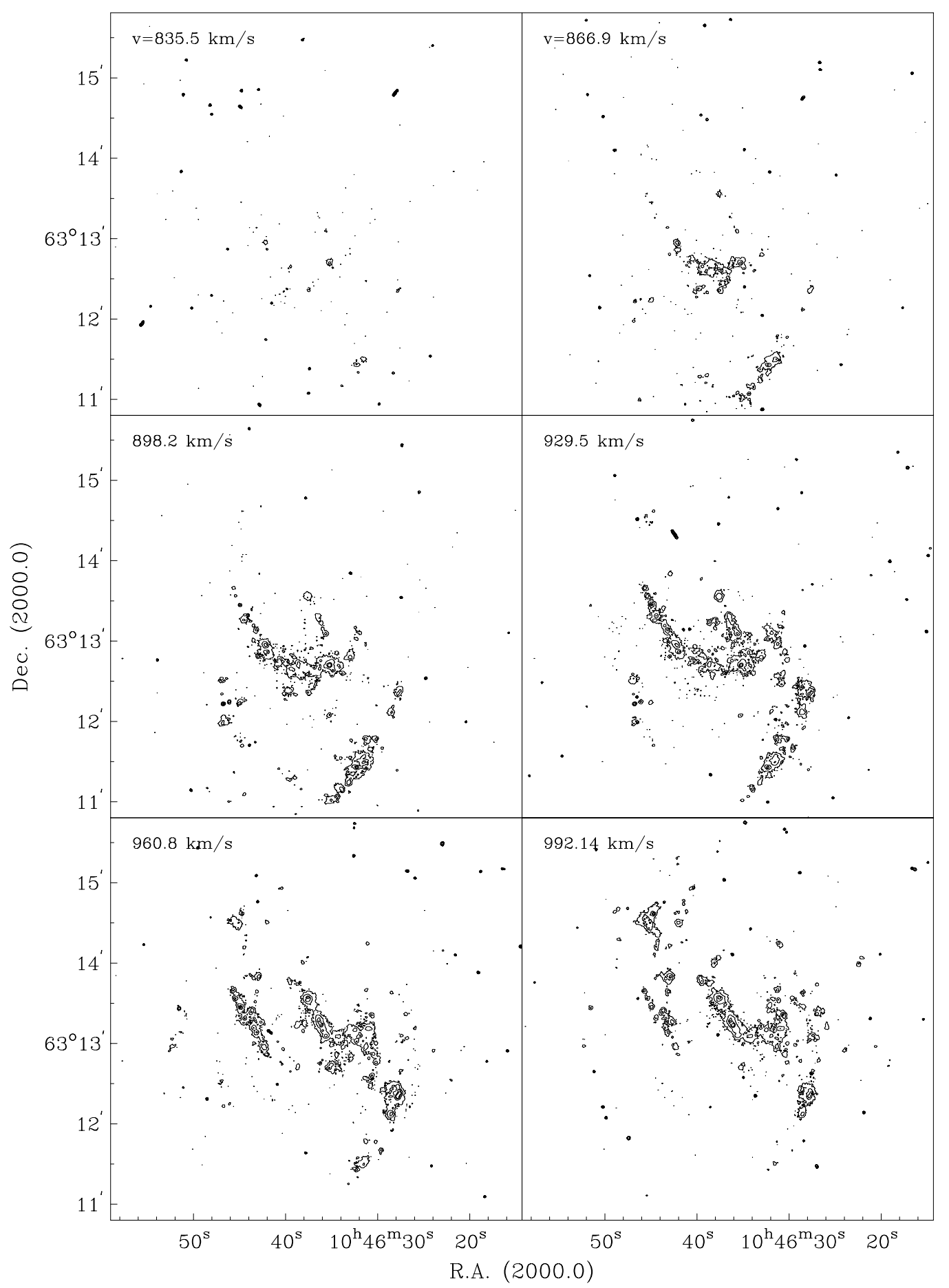

Fig. 1. Planes of the $\mathrm{H} \alpha$ high resolution (1.5") data cube for NGC 3359 (before "cleaning" the data cube; see text for details) at a series of velocities about the central systemic value of $1008 \mathrm{~km} \mathrm{~s}^{-1}$

This was done interactively by inspecting the (high resolution) channel maps one by one, continually comparing with the same channel and referring to adjacent channels in the smoothed cube $\left(6^{\prime \prime}\right)$. Previously we had followed an analogous routine for "cleaning" the cubes at lower resolution, using the $8^{\prime \prime}$ cube as a reference to clean the $6^{\prime \prime}$ cube, and the $16^{\prime \prime}$ cube to clean that at $10^{\prime \prime}$ resolution.
The emission in $\mathrm{H} \alpha$ as a function of wavelength, i.e. of velocity, for the high resolution cube is shown in Fig. 1 in the form of a set of channel maps, again following radioastronomical usage.

Finally the obtained data cubes were used to construct the moment maps (intensity, velocity and velocity dispersion) in the way explained above. The high resolution 


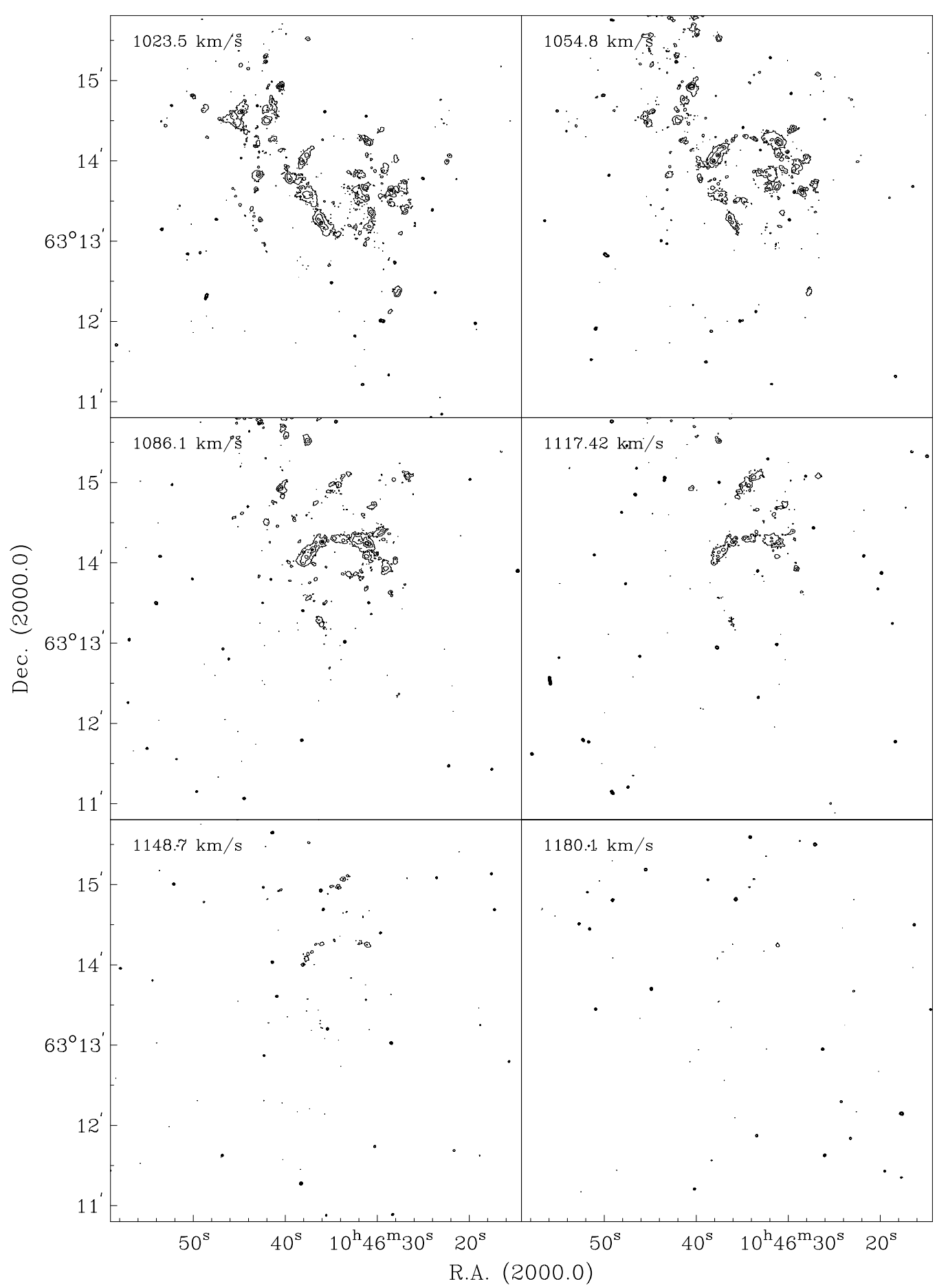

Fig. 1. continued

$\left(1.5^{\prime \prime}\right)$ intensity and velocity maps are shown in Figs. 2 and 3 respectively. These maps could be obtained not only by calculating moments, but also fitting each spectrum to a Gaussian function. The velocity maps obtained with both methods are essentially the same, but considerable differences could appear in the intensity maps and especially in the velocity dispersion maps. This happens because when moments are calculated, it is possible to lose information about the wings of the lines specially if these are low intensity and high velocity dispersion lines (van der Kruit \& Shostak 1982). This fact is due to the selected threshold (normally a fixed multiple of the measured rms noise) bellow which the data is neglected. For Gaussian profiles, the threshold is applied to the line amplitude and not as a fixed value over the noise. This implies that the fit can take into account all the spectrum, but this method is 


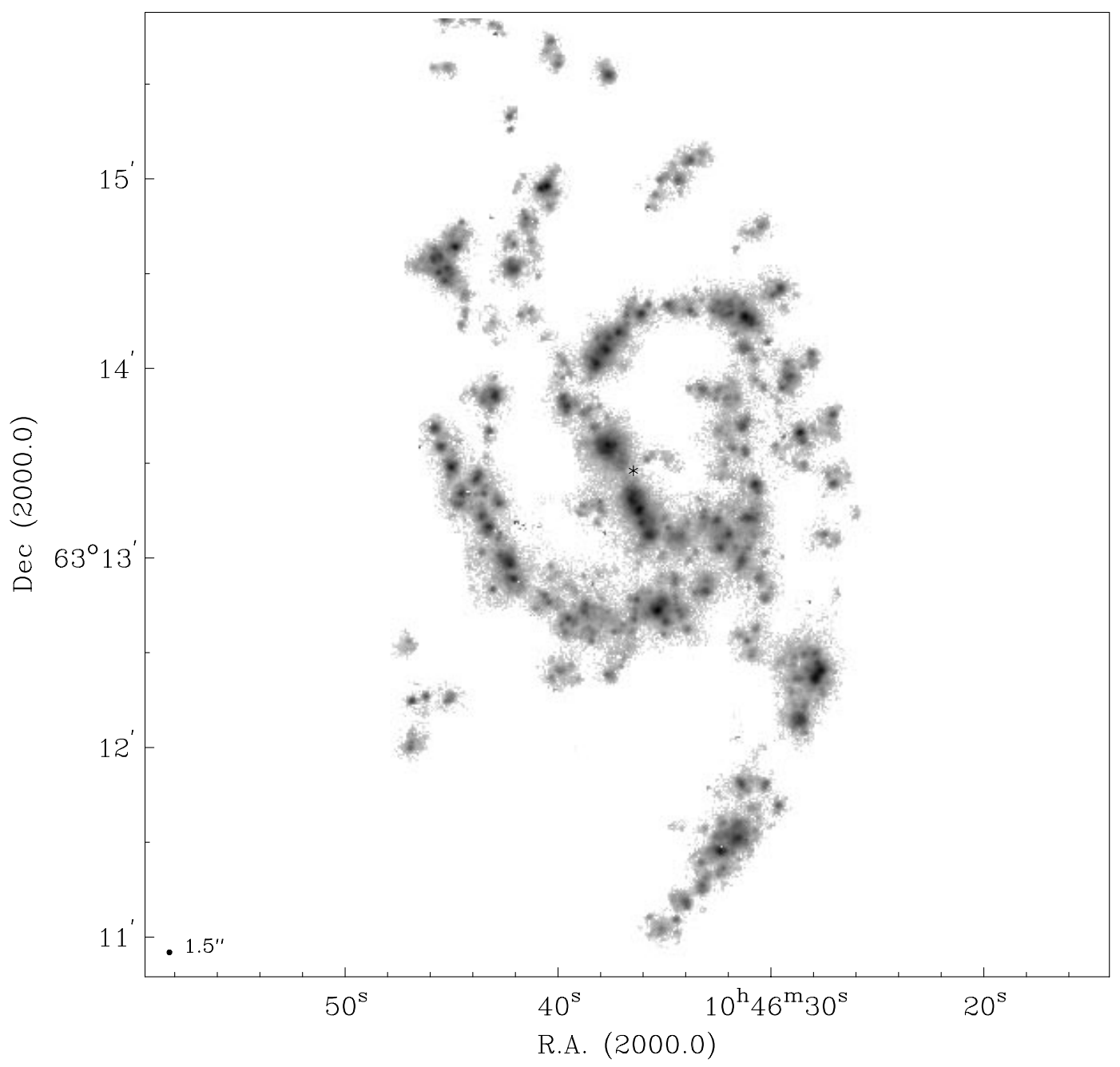

Fig. 2. Intensity map (zeroth moment) of the H $\alpha$ emission in NGC 3359 obtained with the high resolution $\left(1.5^{\prime \prime}\right)$ H $\alpha$ data cube. The dynamical centre is marked with an asterisk

susceptible to error (in the low signal points) because we are considering noisy areas as signal. In this work, we have chosen the moment method. We are aware of the possible effect on the results that we present here, and the analysis of the velocity dispersion map constructed using both methods, a study of all the velocity components of the emission line, and the relation between the principal velocity components with the HiI region luminosity, will be presented in Zurita et al. (2000).

\section{Kinematics}

In the $\mathrm{H} \alpha$ intensity map in Fig. 2 we can pick out the brightest HiI regions, which are also visible in the channel maps (Fig. 1), which do not show special features, apart from those associated with the normal rotation pattern of the galaxy. In the rest of this section we will study in detail the velocity and velocity dispersion maps, and derive the rotation curve.

\subsection{The velocity map}

The velocity map at the highest angular resolution $\left(1.5^{\prime \prime}\right)$ is shown in Fig. 3, calculated as described above using the MOMENTS program in GIPSY. In this map we show the overall velocity field in HII emission: the first moment of the $\mathrm{H} \alpha$ emission for each pixel with measurable emission. 


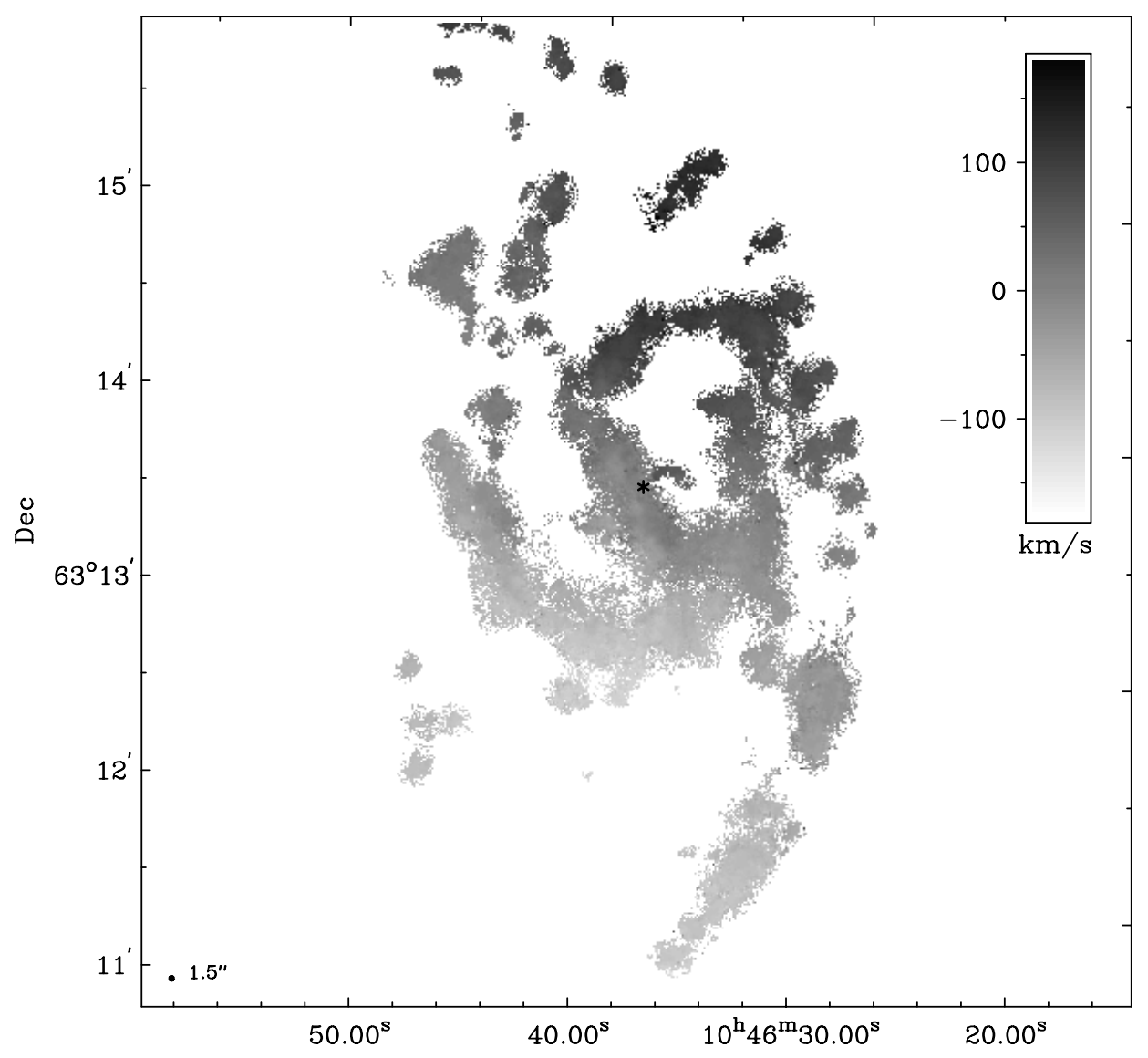

Fig. 3. High resolution $\left(1.5^{\prime \prime}\right)$ velocity map for NGC 3359. The darkest zone is the receding side (positive redshift) and the brightest part is the approaching side of the galaxy. The dynamical centre is marked with a asterisk

In practice, the global kinematics of the galaxy can be seen more clearly in the map smoothed to $16^{\prime \prime}$ resolution shown in Fig. 4, in which isolevels are overlaid on a gray scale representation. The velocity field is generally quite regular although there are some deviations which we will deal with below. In a number of positions on the map, we can detect kinks in the otherwise regular contours, which are recognizable as due to streaming motions related to the behaviour of a density wave in the region of a spiral arm. From the deviations in the isolevels we can estimate amplitudes along the line of sight for these motions, which correspond to $\sim 50 \mathrm{~km} \mathrm{~s}^{-1}$ in the plane of the galaxy disc, deprojected using an inclination angle of $53^{\circ}$.

Although these values are quite high, they are not outside the range of values found in other galaxies, (Visser 1980 for M 81; Rots 1990 for M 51; Knapen et al. 1999 for M 100 and Knapen 1997 for NGC 3631). As well as these deviations in the external part of the disc there are others of greater amplitude which dominate the internal part of the disc and which can reach values as high as $35-40 \mathrm{~km} \mathrm{~s}^{-1}$ projected along the line of sight. Below we show that these are due to non-circular motions around the bar. Ball (1986) found such motions in $\mathrm{HI}$ in the same zone.

The velocity field shows also an interesting feature in the immediate NW and SE of the centre of the bar, which are twin peaks in the $\mathrm{H} \alpha$ emission of the galaxy. However the residual velocities in these peaks do not have identical projected values (in the plane of the sky); in the NW peak the residual velocities reach $45 \mathrm{~km} \mathrm{~s}^{-1}$, but $25 \mathrm{~km} \mathrm{~s}^{-1}$ is the maximum residual value in the peak located in the SE. This feature does not fit the global disc kinematics and it may be due to a velocity component not in alignment with the bar, even perpendicular to it. A study of the dynamical properties of NGC 3359 will include a more detailed study of this feature (Sempere \& Rozas 2000). 


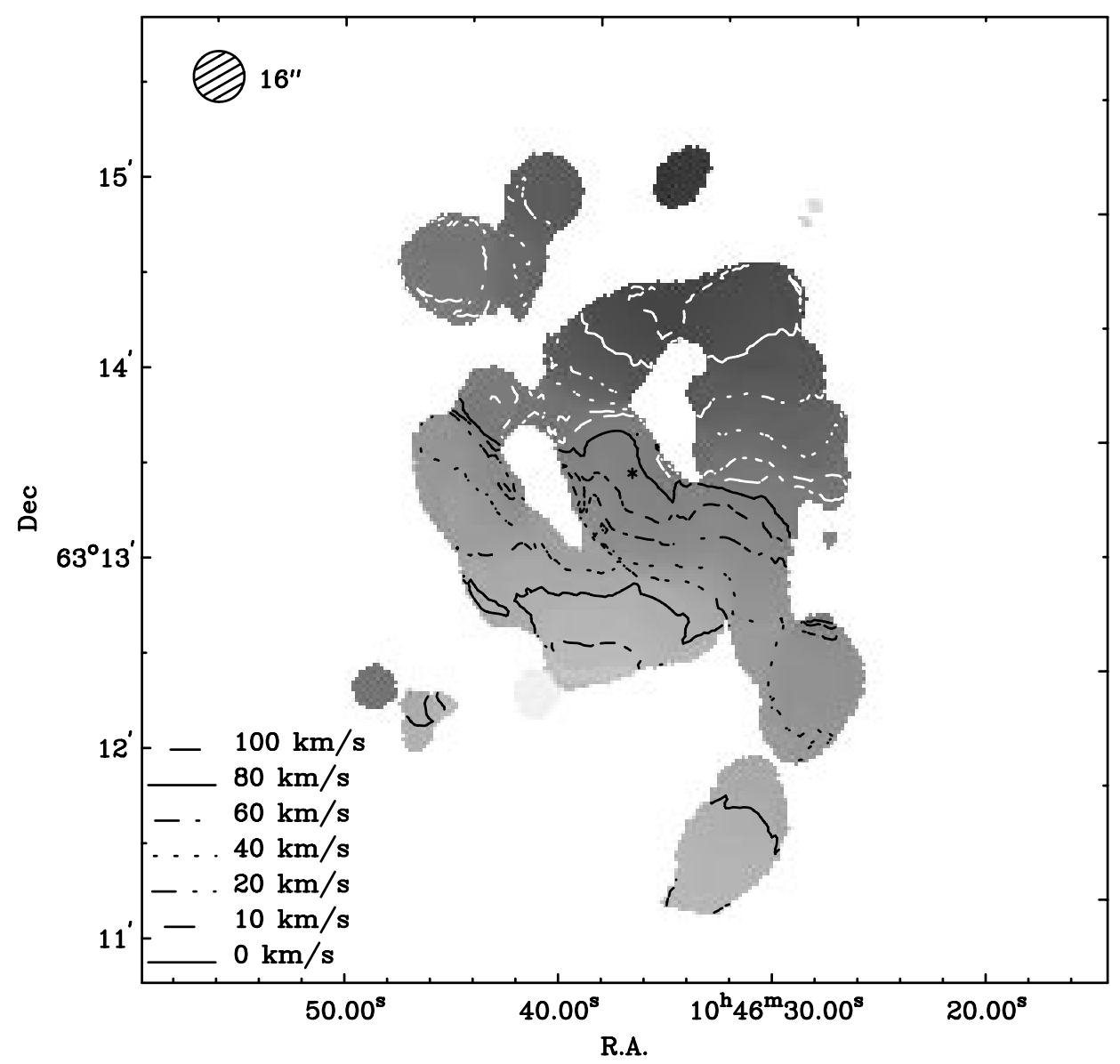

Fig. 4. Low resolution velocity map for NGC $3359\left(16^{\prime \prime}\right)$ presenting more clearly the global kinematics of the galaxy. The dynamical centre is marked with an asterisk and isovelocity contours are overlaid on the velocity map. See the text for details of how the map was produced

\subsection{The rotation curve}

To obtain the rotation curve we use the velocity maps at different angular resolutions, following the procedure described by Begeman (1989), in which the galaxy is divided into a series of concentric elliptical annuli, each described by a set of parameters: $i$ (inclination angle), PA (position angle of the major axis), and $V_{\mathrm{c}}$ (rotational velocity). Additional parameters include the systemic velocity of the galaxy, $V_{\text {sys }}$, and the coordinates of the centre of each annulus: $x_{\mathrm{c}}$ and $y_{\mathrm{c}}$. These parameters are then fitted using a least squares algorithm, which uses the function:

$V=V_{\text {sys }}+V_{\mathrm{c}} \cos (\theta) \cos (i)$

where $\theta$ is the azimuthal angle in the plane of the galaxy measured from the major axis. The points within each annulus are weighted by $\cos (\theta)$, and we eliminated all the points within $15^{\circ}$ of the minor axis, since the errors in deprojecting these points onto the major axis are unacceptable.
In the first instance, to obtain the curve we used the maps smoothed to low resolution $\left(16^{\prime \prime}\right.$ and $\left.10^{\prime \prime}\right)$ to fit the position of the kinematic centre (as this depends only on the symmetry of the velocity field), using in this procedure fixed values of $i=53^{\circ}, \mathrm{PA}=-10^{\circ}$, and $V_{\text {sys }}=1008 \mathrm{~km} \mathrm{~s}^{-1}$ (all the values taken from RC3). The results were: R.A. $(2000)=10^{\mathrm{h}} 46^{\mathrm{m}} 35.55^{\mathrm{s}} \quad( \pm 0.02 \mathrm{~s})$ and dec. $(2000)=63^{\circ} 13^{\prime} 26.1^{\prime \prime}\left( \pm 0.3^{\prime \prime}\right)$. This position was defined using the annuli of radius bigger than $40^{\prime \prime}$, in which the number of independent points for a fit was large, giving rise to small errors in the fit. The kinematic centre coincides with the optical centre in R.A. but there is a shift in dec. of $4^{\prime \prime}$.

The second step was a fine tuning of the system velocity of the galaxy. To do this, we fixed the position of the kinematic centre, found above, and the inclination angle, deriving a satisfactory value for the systemic velocity of $1006.8( \pm 0.3) \mathrm{km} \mathrm{s}^{-1}$, very similar to the value found by 


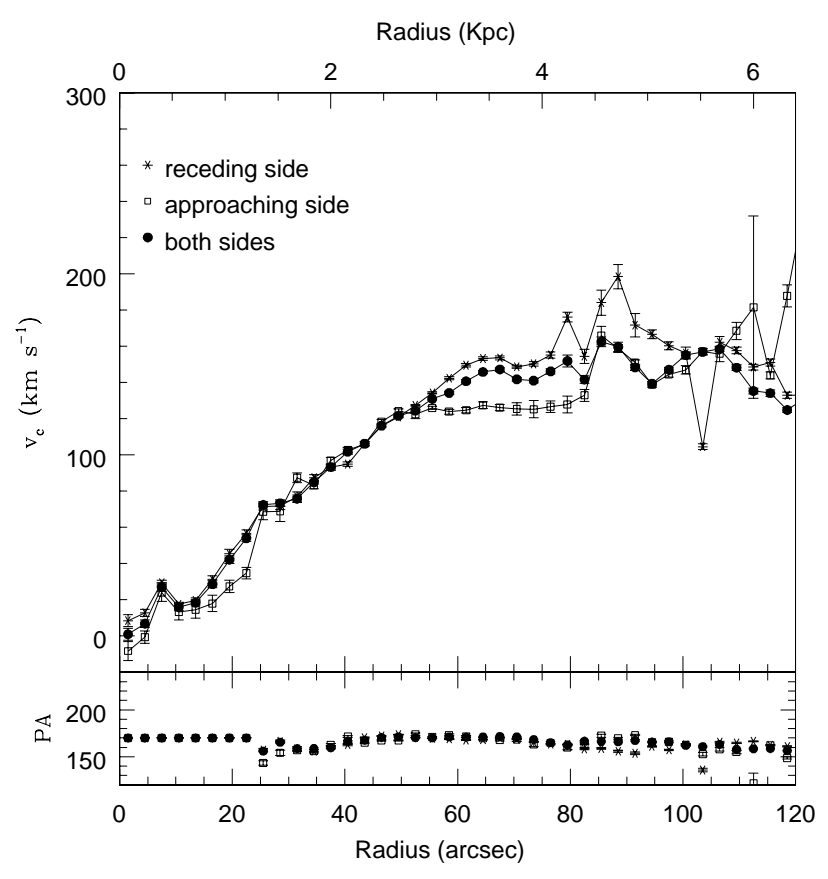

Fig. 5. Rotation curve (obtained from the $6^{\prime \prime}$ resolution data cube) for NGC 3359 from $\mathrm{H} \alpha$ velocities (upper graph) and computed major axis position angle (lower graph)

Ball (1986) from HI, of $1009 \mathrm{~km} \mathrm{~s}^{-1}$, and to the previous optical determination of $1008 \mathrm{~km} \mathrm{~s}^{-1}$ given in RC3.

As an initial choice, we use the inclination angle of $53^{\circ}$ found in the RC3 catalogue. Since the inclination of the galaxy is not especially low, we made a series of test fits using sets of values of $i$ and $V_{\mathrm{c}}$, and finding inclination angles between $48^{\circ}$ and $58^{\circ}$. The best fits, in fact, coincided with the value of $53^{\circ}$ given in RC3, in fair agreement with those used by Ball (1986) and Gottesman (1982) of $51^{\circ}$.

As there are no signs of a warp in this galaxy, we can use a constant value of $i$ for the whole disc; in any case the effect of using a different value is not to change the shape of the rotation curve but to rescale the values of $V_{\mathrm{c}}$. Using the value of $i=53^{\circ}$, we find values for $V_{\mathrm{c}}$ at large galactocentric radii of $145 \mathrm{~km} \mathrm{~s}^{-1}$, which fits within the range of values of the synthetic curves produced by Rubin et al. (1985) for a galaxy of this morphological type and luminosity.

We then used the same low resolution $\left(16^{\prime \prime}\right)$ map to derive an initial rotation curve: the values of $V_{\mathrm{c}}$ and $\mathrm{PA}$ fits for each radius using a radial interval of $8^{\prime \prime}$ between annuli. Afterwards rotation curves were derived for the whole disc, and separately for the approaching and receding halves, using the maps at different resolutions, and employing widths for the annuli of half the resolution limit for the corresponding map. Following the same procedure as for the low resolution map, we checked the values for the kinematic centre position and the systemic velocity,

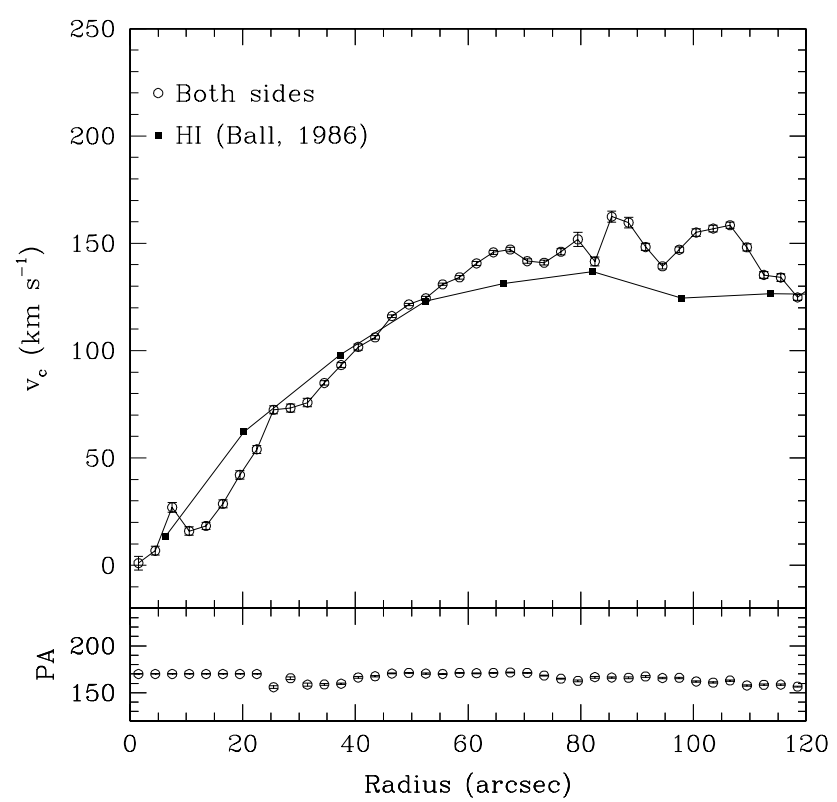

Fig. 6. Rotation curve for NGC 3359 derived in the present study superposed on that obtained by Ball (1986) from HI observations

finding the same values as before, and then adjusted the values of $V_{\mathrm{c}}$ and $\mathrm{PA}$ for each of the higher resolution cubes. The final rotation curve (obtained with the 6 " resolution map) is shown in Fig. 5, and was obtained after a full analysis to check the effects of fixing or freeing the position angles of the annuli and their widths, and to see what would be the effect of including expansion motion of any of the rings in Eq. (1). The definitive curve was then taken by setting the expansion velocity in the disc to zero, and with annuli of $3^{\prime \prime}$ width. The position angle was fixed for radii of less than $20^{\prime \prime}$, (to avoid the effects of the relatively strong non-circular motions in this zone) and free at larger radii.

The upper panel of Fig. 5 shows the rotation curve for the whole disc, and separately for the approaching and receding halves, while the lower panel shows the position angle of the major axis $v$. radius, measured from North through East. Out to a radius of $50^{\prime \prime}$ we see the clear coincidence between the curves for the blue-shifted and redshifted sides of the galaxy, showing that the position of the kinematic-dynamic centre is valid. Out to this radius the curve can be described as that of rigid body motion, with $V_{\mathrm{c}}=a+b r$, where $a=-1 \pm 4$, and $b=142 \pm$ $6 \mathrm{~km} \mathrm{~s}^{-1} \operatorname{arcmin}^{-1}$. The curve is reliable out to $80-90^{\prime \prime}$ radius; beyond this range, the paucity of points available to make a fit means that detailed features of the curve may not be valid, but the general trend agrees well with that derived from Hi by Ball (1986), and the result is shown 


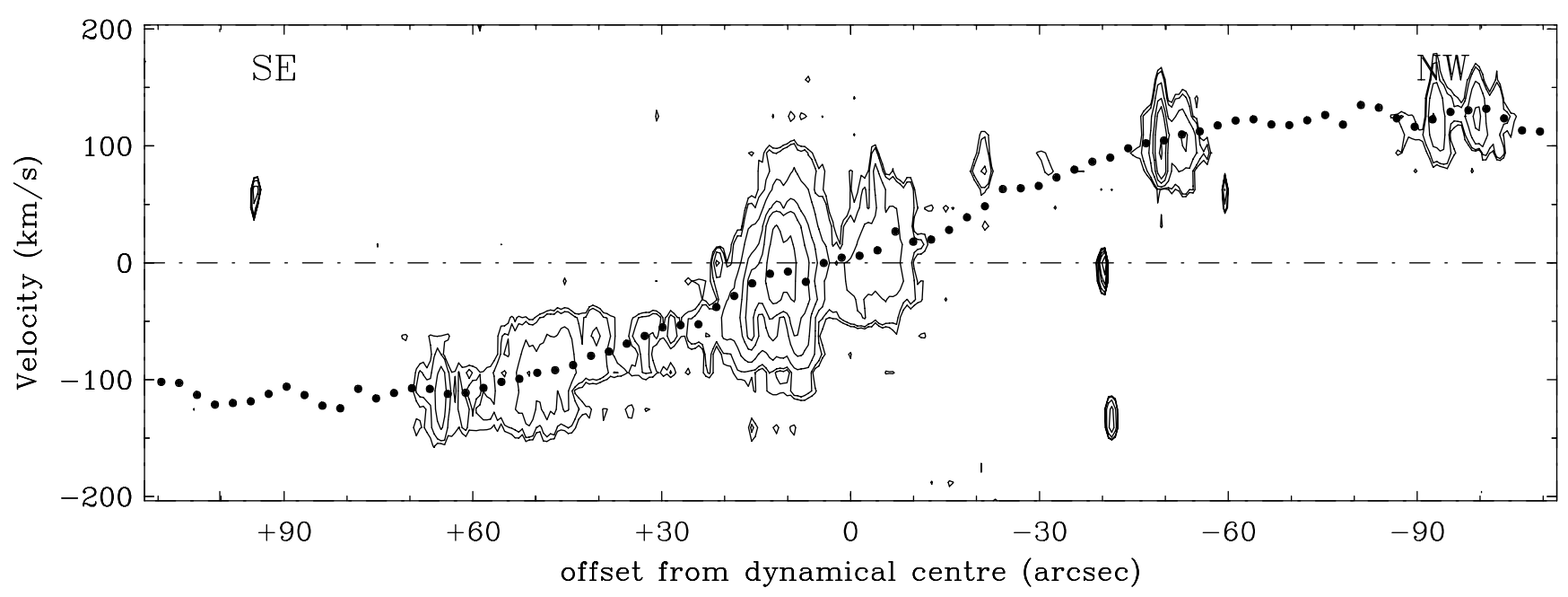

Fig. 7. Position-velocity diagram along the major axis of NGC 3359. Superposed is the inner portion of our computed mean rotation curve

in Fig. 6. Although the two curves are similar, we note that our curve shows, on average, values $10 \mathrm{~km} \mathrm{~s}^{-1}$ higher than the Hi curve in the radial range beyond 50" from the centre. In Fig. 7 we show a position-velocity diagram along the major axis. In this figure we have superposed the inner part of the mean rotation curve and the good coincidence between this curve and the gas distribution shown indicates that the curve derived is reasonable.

\subsection{Model velocity map, and residual velocity field}

The derived values of $V_{\mathrm{c}}$ and PA have been used to construct an axisymmetric model of the velocity field, with the remaining parameters as specified above: $\left(i, V_{\mathrm{sys}}, x_{\mathrm{c}}, y_{\mathrm{c}}\right)$. The most external parts of the model velocity field (beyong $80^{\prime \prime}-90^{\prime \prime}$ ) have been calculated using the velocity value to which the rotation curve tends at large galactocentric radii. The model is shown in Fig. 8. The strongest deviations from regularity, in a clockwise direction could be artificial, due to a point with a value of PA lower than the true value. The model is quite smooth, though the deviations due to streaming motions across the arms can be see at $\sim 1.5^{\prime}$ in from the kinematical centre.

By subtracting the two-dimensional model of the regular velocity field from the observed field we obtain a map of the residual velocity field which is shown in Fig. 9. This map allows us to study the global field of the non-circular components of the motion of the IS gas, which can be either radial or vertical (only by using external information can we try to distinguish between these cases, though this information does exist for key features of the galaxy). The largest residuals coincide with the bar, along which there is a clear velocity gradient. The residuals attain values of up to $35 \mathrm{~km} \mathrm{~s}^{-1}$ along the line of sight. This velocity gradient represents a real dynamically induced field of motion in the ionized gas, and is not an artifact of the model subtraction. We have derived residual maps using a range of possible fits to the rotation curve on which the central zone of the model is based, and all of these give rise to the same form of residual field around the bar.

In the disc, the residual velocity field shows a zone of negative values close to the minor axis. This could be due to the exclusion of those points within a $15^{\circ}$ angle of the minor axis from the set used to compute the rotation curve. In the residual velocity map we can pick out the spiral arms by the positive residuals due to the streaming motions which we could also identify in the model field. Finally some of the HiI regions show a notable residual velocity signature due to their high internal velocity dispersions, (see the next section). These peaks of residual velocity are evidence for expansive motions associated with each of the regions concerned. In certain dynamical models of the most active star forming regions, containing $\mathrm{O}$ and B stars, motions of this type, giving rise to "chimneys" were proposed by Norman \& Ikeuchi (1989).

\subsection{The velocity dispersion map}

The velocity dispersion is the last parameter whose behaviour is described from one of the moment maps of the disc of NGC 3359: in this case the second moment. The original map was obtained using the MOMENTS program 
in GIPSY, with the values of the Gaussian widths of the emission lines fitted in each pixel. The velocity dispersion map in Fig. 10 is not directly taken from the observations. It has been corrected for the instrumental width, the natural line width, and the thermal line width at each point. These widths must be subtracted in quadrature from the observed width, $\sigma_{\mathrm{obs}}$, to yield the width due to the mean internal motions of each region, which we assume to be dominated by turbulence. The expression used in this subtraction is:

$\sigma_{\text {obs }}=\left[\sigma_{\mathrm{N}}^{2}+\sigma_{\text {Ins }}^{2}+\sigma_{\mathrm{t}}^{2}+\sigma_{\mathrm{nt}}^{2}\right]^{1 / 2}$

where the natural, instrumental, and thermal widths are termed $\sigma_{\mathrm{N}}, \sigma_{\text {Ins }}$ and $\sigma_{\mathrm{t}}$, respectively.

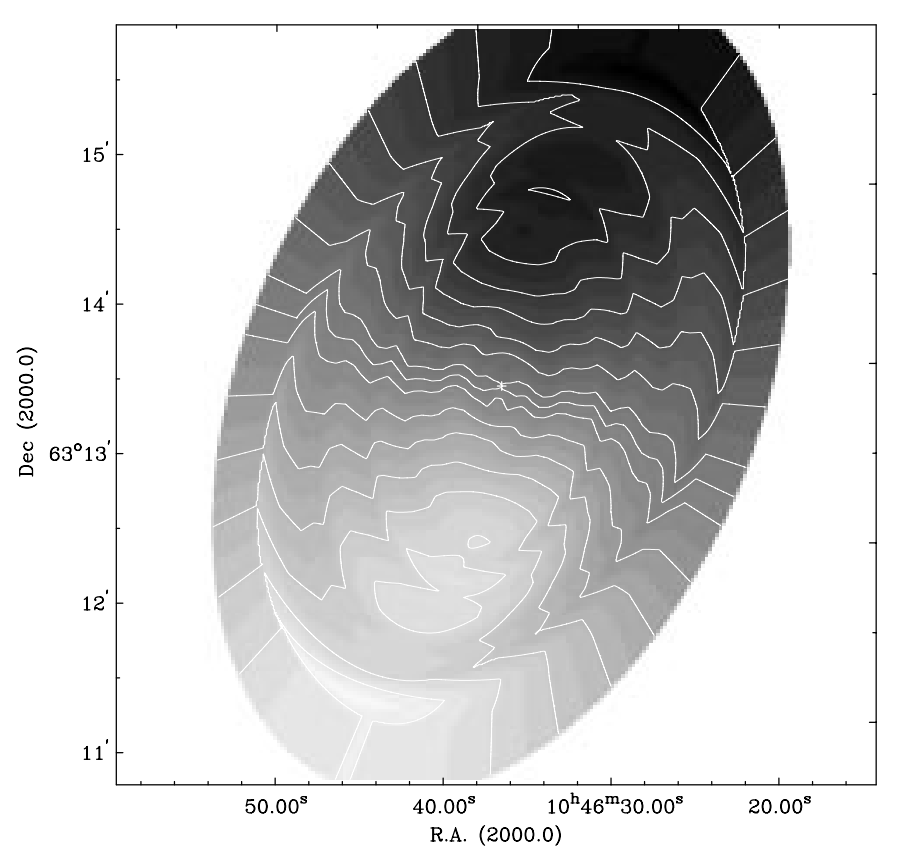

Fig. 8. Model rotational velocity map of NGC 3359, computed using fits to a set of inclined annuli (see text for more details). The isolevels have a separation of $10 \mathrm{~km} \mathrm{~s}^{-1}$

The natural width is virtually constant for hydrogen, taking a value of $0.16 \AA$, which is the equivalent of $3 \mathrm{~km}$ $\mathrm{s}^{-1}$ (O'Dell \& Townsley 1988). The instrumental width was calculated knowing the intrinsic width of the laboratory source which was used to produce the calibration cube. After close analysis of this cube we showed that there were no systematic structures due to calibration anomalies, and that the instrumental dispersion map showed a constant width of $16.2 \pm 0.3 \mathrm{~km} \mathrm{~s}^{-1}$, which we used for $\sigma_{\text {Ins }}$. The thermal width, $\sigma_{\mathrm{t}}$ was taken by assuming a temperature of $10^{4} \mathrm{~K}$ for the ionized gas (Spitzer 1978; Osterbrock 1989), and is $9.1 \mathrm{~km} \mathrm{~s}^{-1}$.

In the velocity dispersion map we can see that the highest dispersions correspond to the most luminous HII regions, and this gives rise to a correlation between the dispersion map and the map of residuals. A more detailed analysis will be needed to quantify these relations, (Zurita et al. 2000, in preparation) but if they are similar to those found in M 100 and M 101 (Rozas et al. 1998a) as we predict from a quick look, we will have further evidence for density bounding in these regions. The mode value for the dispersions in the galaxy is close to $15 \mathrm{~km} \mathrm{~s}^{-1}$, as we can see if Fig. 11. This value remains rather constant outside a radius of $30^{\prime \prime}$. There are several possible heating mechanisms which might underly this behaviour, including the conversion of galactic rotational energy to random cloud-cloud dispersion through viscosity (Combes \& Becquaert 1997). At smaller galactocentric distances the greater concentration of star formation would imply that this source is the dominant heating mechanism.

\section{The bar of NGC 3359}

The bar of NGC 3359 has a deprojected total length of 2.9 arcmin (which corresponds to $\sim 9 \mathrm{kpc}$ ). Around $17 \%$ of the total $\mathrm{H} \alpha$ flux from the galaxy comes from the bar, which exhibits a string of very bright, prominent HiI regions, or "hotspots".

According to Friedli \& Benz (1993) and to Friedli et al. (1994) strong flows of gas are predictable along a bar which is at a formative stage. The hotspots in this case can be explained as due to the star formation in the gas which has not contributed to the intense star formation at the ends of the bar (due to interstellar shocks) but falls towards the centre as its angular momentum is absorbed in the shocks and in the more general bar potential. This gas can be trapped in the inner resonant structure of the galaxy, producing massive stars and hence the hotspots.

Properties of bars related to their velocity structures include their metallicity gradients. In a general study of metallicity gradients in galaxies, Vila Costas \& Edmunds (1992) showed that the gradients within the bars of barred galaxies are much lower (almost zero) than those in the external discs. In NGC 3359, however, Martin \& Roy (1995) found a higher gradient within the bar, using oxygen as the abundance probe. They attributed this effect to the fact that in NGC 3359 the bar is still forming, so that there has not been time for turbulence to smooth out the intrinsic gradient due to a radially differential star formation rate. A related effect of this type of mixing in the disc is the reduced star formation symmetry in the arms of barred spirals compared with non-barred spirals, as reported in Rozas et al. (1998b).

In our residual velocity map, the zone around the bar is noticeable for the high values of its residuals $(v>$ $30 \mathrm{~km} \mathrm{~s}^{-1}$ ) found after subtracting off the rotational model. In Fig. 12 we show an integrated profile along the bar (with a integration size of 5 pixels, which represents $2.8^{\prime \prime}$, which clearly shows the amplitude of the residuals, 


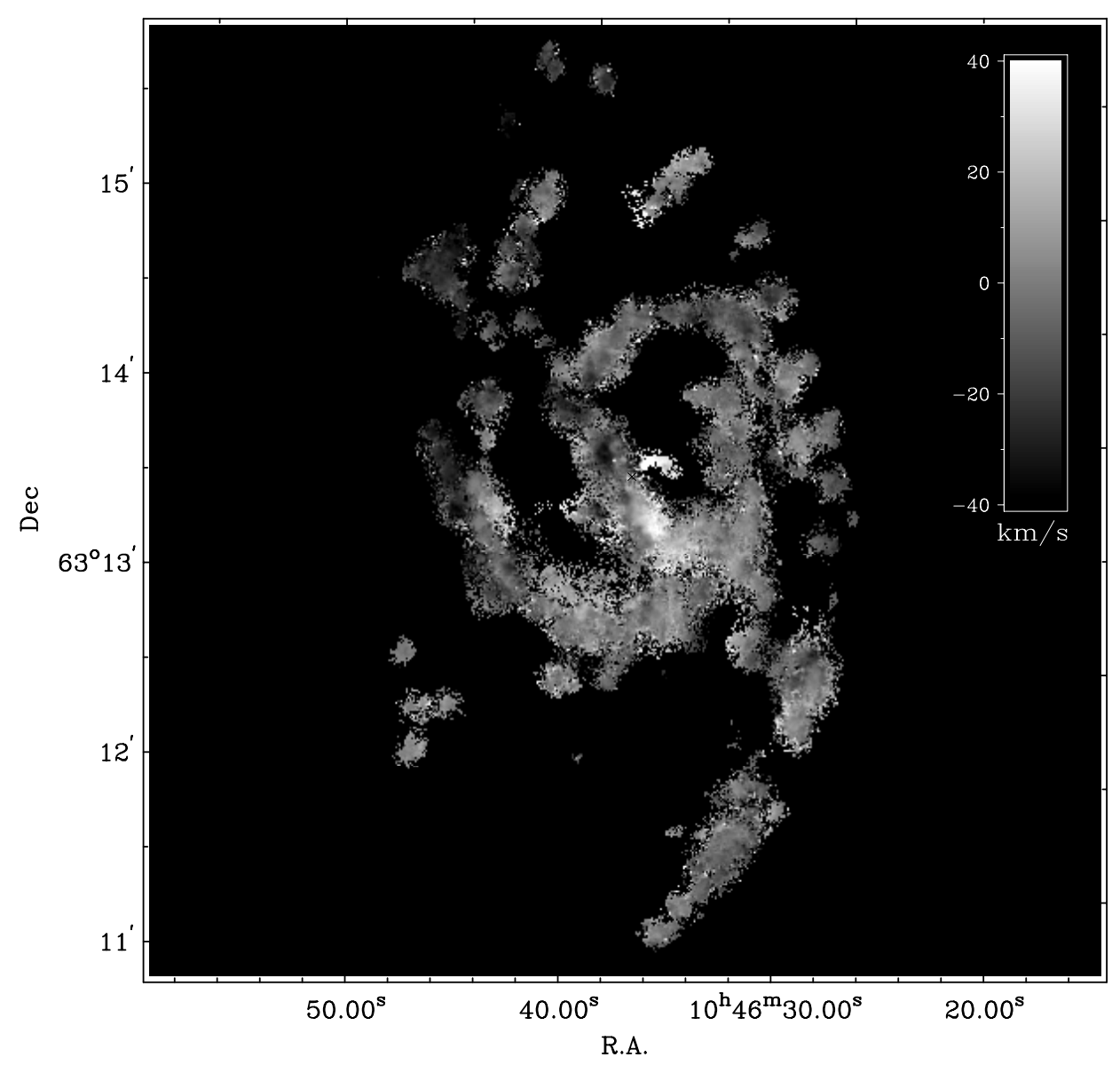

Fig. 9. Map of residual velocities for NGC 3359 obtained by subtracting off the two-dimensional model computed from the rotation curve from the observed velocity map. The resolution is $1.5^{\prime \prime}$

(up to $30 \mathrm{~km} \mathrm{~s}^{-1}$ and $35 \mathrm{~km} \mathrm{~s}^{-1}$ in the NE and $\mathrm{SW}$ zones respectively) and their symmetry with respect to the bar centre.

These residuals are also detected in cuts made along and perpendicular to the $\mathrm{H} \alpha$ bar (PA 28 $8^{\circ}$ through the data in the original cube, and they are much too strong to be artifacts. The positions of these cuts are indicated with a diagram in Fig. 13.

We show the velocities along the perpendicular cuts in Fig. 14. In the graph, in the upper left hand corner we see the distance measured from the dynamical centre, positive to the NE, negative to the SW. Given the orientation of the bar with respect to the major axis, these cuts should show the projection of the rotation curve in the direction of the bar. In the upper panels, to the $\mathrm{N}$ of the dynamical centre (see Fig. 13), we should see positive velocities, and in the lower pannels after the dynamical centre, to the $\mathrm{S}$, negative velocities; however at $+9.6^{\prime \prime} \mathrm{N}$ of the dynamical centre the velocity at peak intensity suddenly goes more negative, and then rises to $0 \mathrm{~km} \mathrm{~s}^{-1}$. This is consistent with the existence of negative residual velocities in the NE section of the bar. From the dynamical centre in the SW direction the opposite effect is seen; between $-9.6^{\prime \prime}$ and $-12.8^{\prime \prime}$ : there is a displacement of the peak emission to positive velocities corresponding to the positive residuals we observe in the map of residuals. These coincide not only in position along the bar, but also in velocity amplitude. One should be aware that the kinks in the countours at $-50 \mathrm{~km} \mathrm{~s}^{-1}$ in the botton 3 panels of Fig. 14, are artificial due to the fact that we used the original data cube to derive the position-velocity diagrams (before "cleaning" the noise in the data cube) in order to preserve the information at low signal levels.

In Fig. 15 we show the cuts parallel to the bar, ordered NW to SE. The most illustrative cut is that which passes through the dynamical centre. In this panel, we show the velocity rotation curve, projected in the direction of the cut (parallel to the bar). We can see how, in the SW of the bar, where the peak intensity shows negative velocities, the intensity contours have offsets to positive 


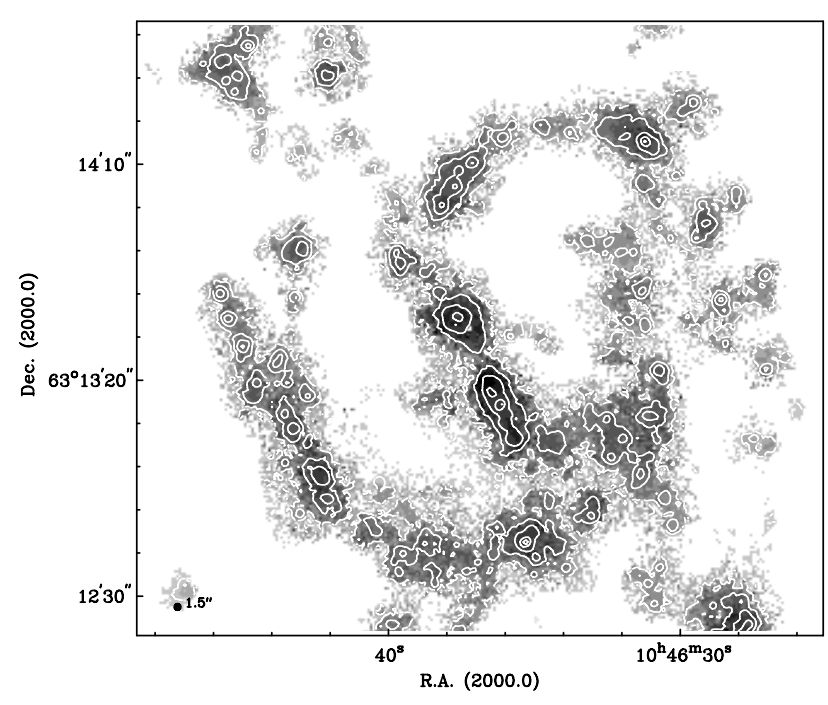

Fig. 10. Map of the velocity dispersion in the $\mathrm{H} \alpha$ emission of NGC 3359, with isointensity contours superposed. Darker shading corresponds to higher velocity dispersions

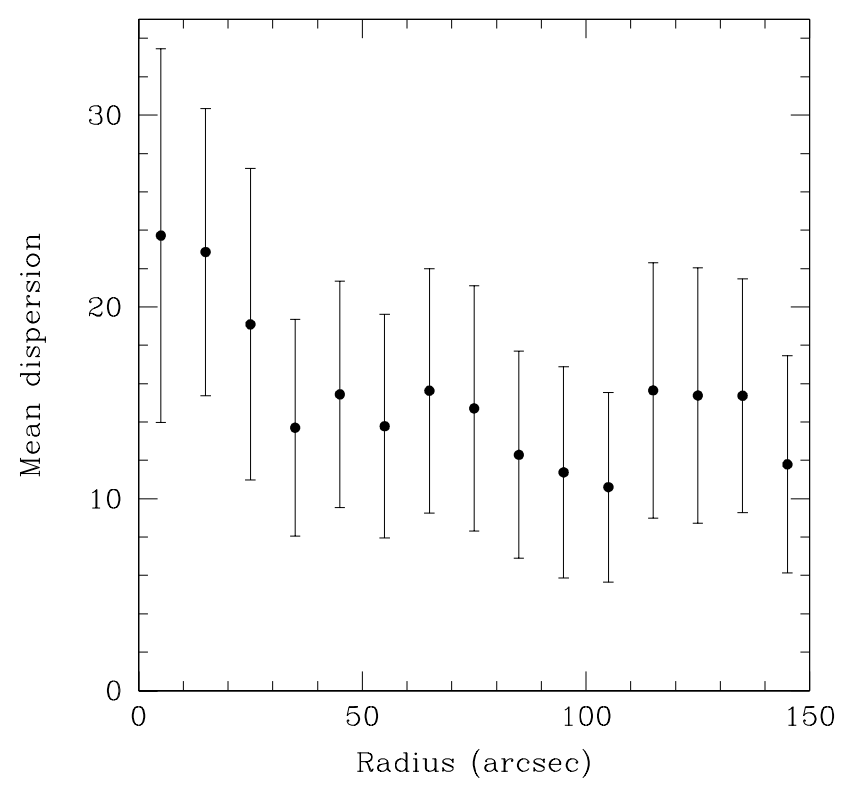

Fig. 11. Radial distribution of the velocity dispersion in annuli of width $8^{\prime \prime}$

velocities. The opposite effect is seen in the NE. These elongations can be readily explained if there are two components of velocity at the corresponding points on the bar. The existence of these components: red-shifted in the SW and blue-shifted in the NE, coincides with a well-defined gradient in the residual velocity field. The cut at $-3.0^{\prime \prime}$

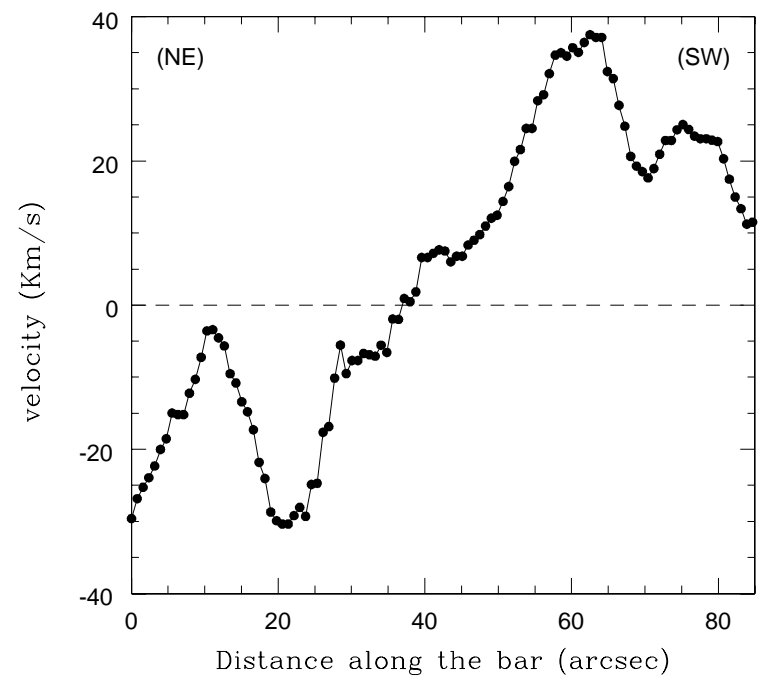

Fig. 12. Integrated profile of the residual velocity of the $\mathrm{H} \alpha$ emission along the bar of NGC 3359

from the dynamical centre is of particular interest; here we are in a direction between the major and minor axes of the galaxy, but even so the maxima in the velocities of the regions have the same radial velocity. The residual non-circular motions are "compensating" the differences in projected velocity which we would expect for a simply rotating disc at these positions.

The velocity gradient observed could well be due to the projection along the line of sight of the velocity of the gasflow round the stellar bar of the galaxy. To determine the orientation of this stellar bar we have used infrared images in $J$ and $K$, the symmetry of whose isophotes yields a position angle of $15^{\circ}$. The outer isophotes in an $I$ band image suggest a value of $7^{\circ}$, used by Ball (1992), but the internal isophotes in this band give a value of $15^{\circ}$, equal to those in $J$ and $K$ (Paper I). Roberts et al. (1979) demonstrated that the presence of a non-axisymmetric potential in a spiral galaxy, of barred form in the inner zone, and spiral in the outer, will give rise to a gas response in the inner part of the disc in the form of non-circular motion along quasielliptical stream-lines. They predict that a strong velocity gradient across the bar can be the result of the highly oval gas circulation driven by the barlike potential field in the inner parts, and predicts too, supersonic velocities at both sides of the bar. Following this predictive model we assumed that the strong velocity gradient observed across the bar, (which is plotted in Fig. 17 as a function of the azimuthal angle in the plane of the galaxy) was due to the projection (in the plane of the sky) of the tangential component of the velocity gas flow (assumed to be in elliptical orbits).

For NGC 3359 we have assumed as a first approximation that the observed residuals are the projection of fluxes of this form, without extra components. We have taken 


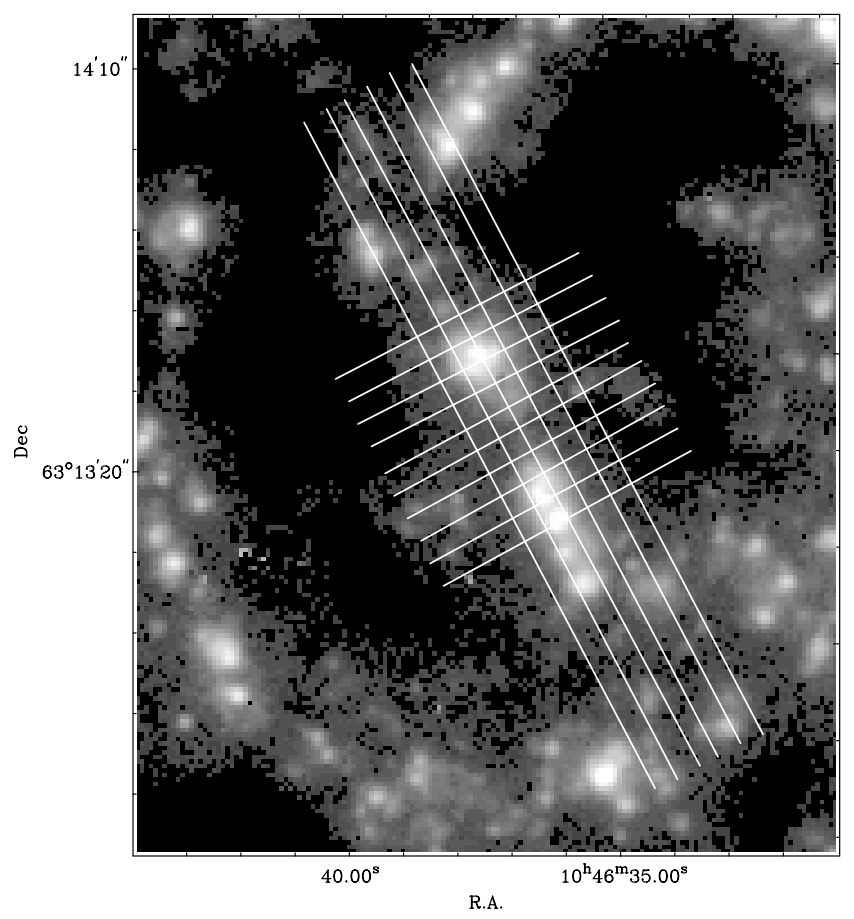

Fig. 13. Diagram showing the cuts made along and perpendicular to the bar over the $\mathrm{H} \alpha$ intensity map of the bar

the orbits of the gas around the bar to follow ellipses with axial ratio $\cos \left(70^{\circ}\right)=0.34$, which is the best fit to the bar isophotes. We integrated the map of residual velocities obtained with the low resolution $\left(16^{\prime \prime}\right)$ data cube, a procedure adopted to overcome the problem posed by the patchy nature of the emission from the ionized gas, using two concentric annuli, centred on the dynamical centre, with $\mathrm{PA}=15^{\circ}$, and effective $i=70^{\circ}$, divided into sectors of $20^{\circ}$ which enabled us to obtain a mean velocity value for each sector (see Fig. 16). With the values of the projected velocities as a function of the azimuthal angle, the inclination angle of the bar and that of the galaxy $\left(53^{\circ}\right)$, we performed a deprojection to infer the in-plane velocities which produce the projected values observed for the external annulus.

The results are shown in Fig. 17. In the upper panel we plot the modulus of the tangential velocity component of the assumed elliptical gas flow, obtained using the mean projected velocity as (middle panel) a function of azimuthal angle in the plane of NGC 3359. The origin of the graph axis is in the NE of the bar, and the angles are measured clockwise. Results show a high degree of symmetry, in the sense that maximum values of the velocity occur at an angular separation of $\sim 180^{\circ}$, which corresponds to either side of the bar, as predicted by Roberts et al. (1979), if the observed velocity gradient is in fact due to the gas flow, caused by the shocks at the ends of the bar. The gas flux shows a velocity gradient across the bar, reach-

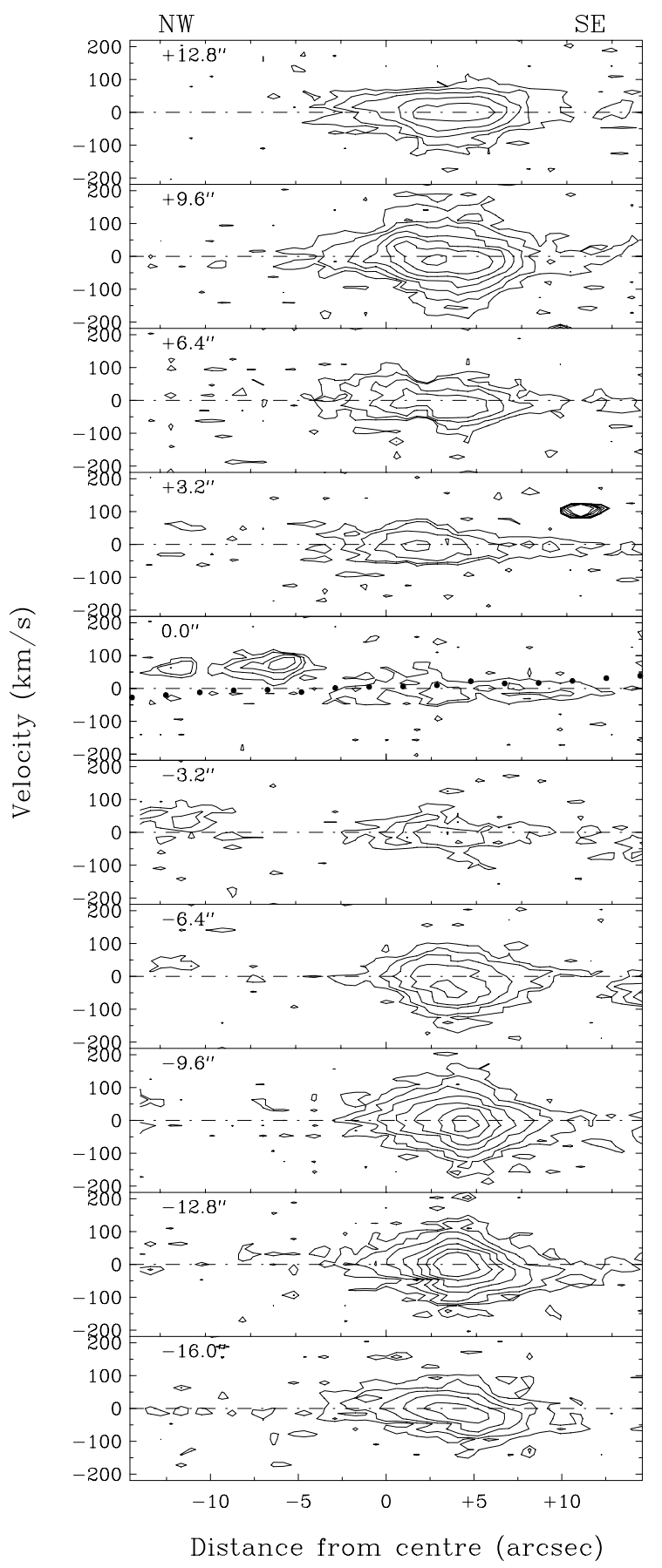

Fig. 14. Position velocity diagrams of the $\mathrm{H} \alpha$ emission perpendicular to the bar of NGC 3359. The rotation curve, projected in the direction of the slice, has been overlaid in the panel of the cut which passes through the dynamical centre

ing maximum values of $40 \mathrm{~km} \mathrm{~s}^{-1}$ for the annulus with width $34^{\prime \prime}$ and major axis $67^{\prime \prime}$ in length. We will confirm this kinematic analysis with the study of other gaseous components (Boonyasait et al. 1999, in preparation). 


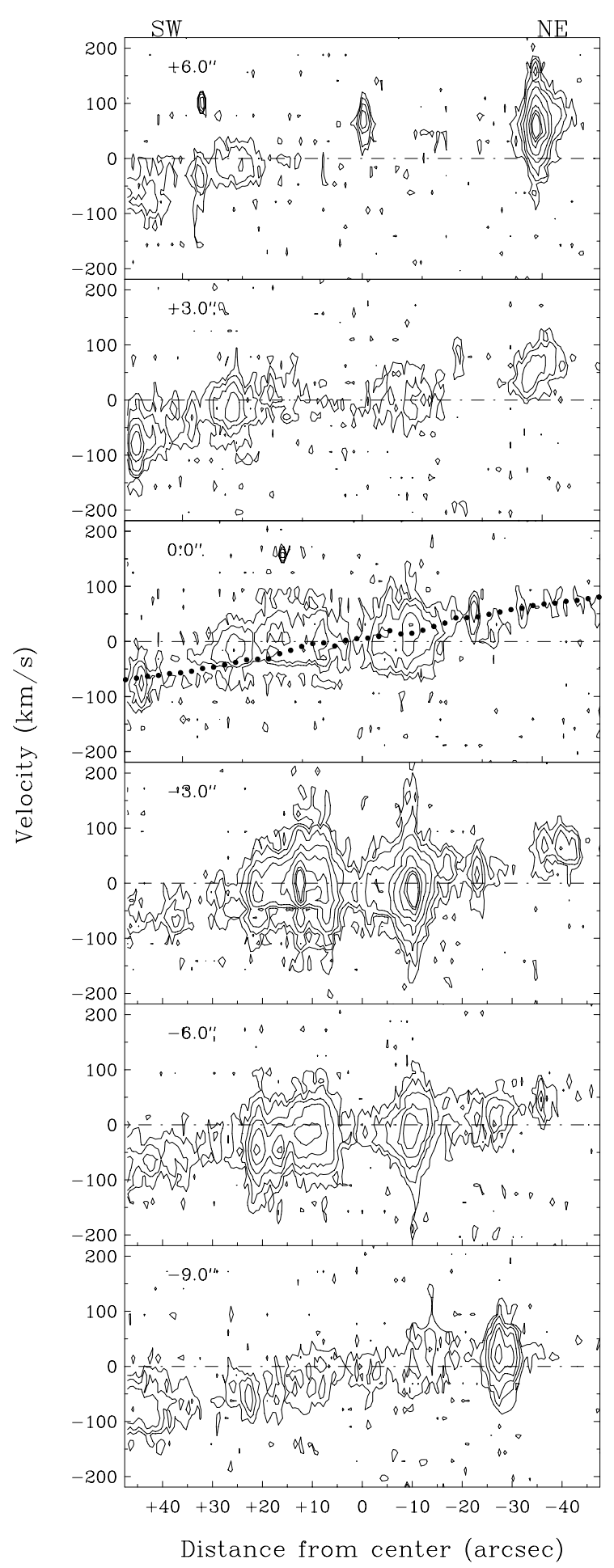

Fig. 15. Position velocity diagrams of the $\mathrm{H} \alpha$ emission parallel to the bar of NGC 3359. The rotation curve, projected in the direction of the slice (parallel to the bar), has been overlaid in the panel of the cut which passes through the dynamical centre

\section{Conclusions}

The main results of the present paper, where we analyze the kinematics of the ionized gas in NGC 3359 are summarized below.

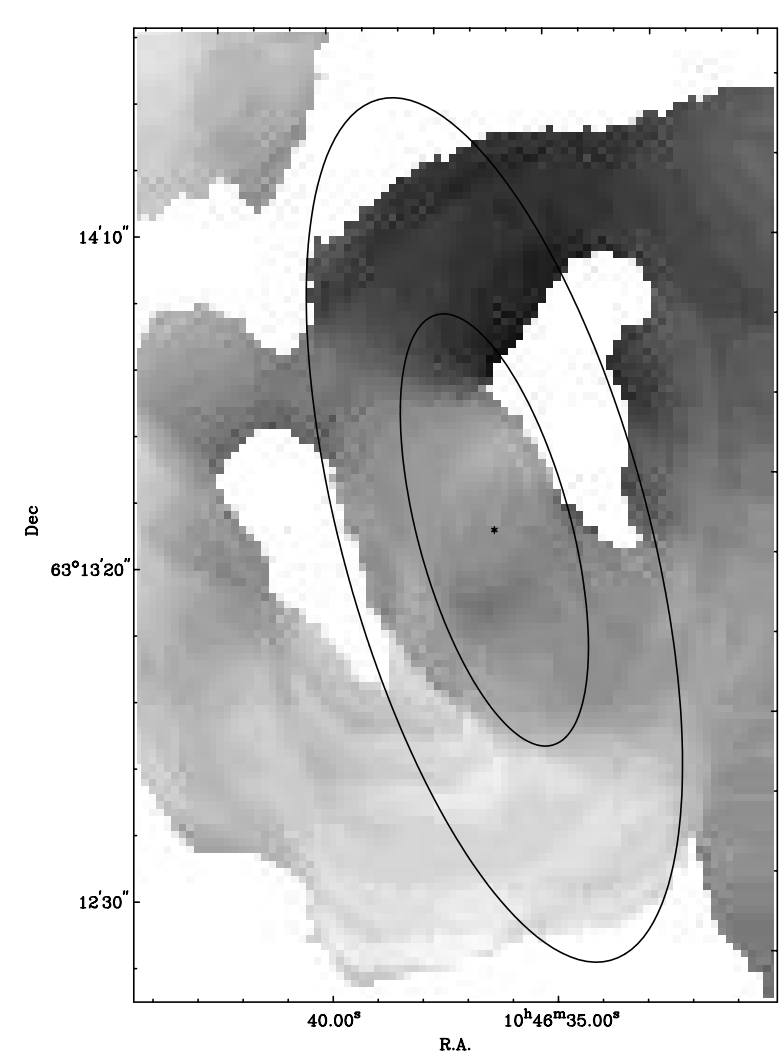

Fig. 16. Low resolution $\left(16^{\prime \prime}\right)$ map of the residuals in the bar zone, with ellipses chosen to conform to the predicted orbits of the gas around the bar

- A velocity map of the ionized gas in the barred spiral NGC 3359 has been produced using Fabry-Perot observations. Analysis of this map shows a disc with an axisymmetric rotation pattern but also evidence of deviations from circular motions in the arms (streaming motions) and near the bar;

- We have calculated from the velocity map the disc kinematic parameters of NGC 3359, which are coincident with those found in the literature for this galaxy: $i=53^{\circ}, V_{\mathrm{sys}}=1006.8 \mathrm{~km} \mathrm{~s}^{-1}$;

- The rotation curve of the galaxy shows a standard shape with an asymptotic rotation velocity of about $145 \mathrm{~km} \mathrm{~s}^{-1}$. Both the rotation curve and the asymptotic rotation velocity are compatible with the previous Hi determinations, although our results have a better spatial resolution;

- We present a map of the velocity residuals, subtracting the axysimetric rotation model from the observed velocity map, which shows prominent peaks in some areas of the disc, indicating non-circular motions of the following three kinds:

1. Streaming motions in the spiral arms;

2. Gas flows around the bar;

3. Vertical motions associated with the turbulent dispersion in the brightest HII regions. 


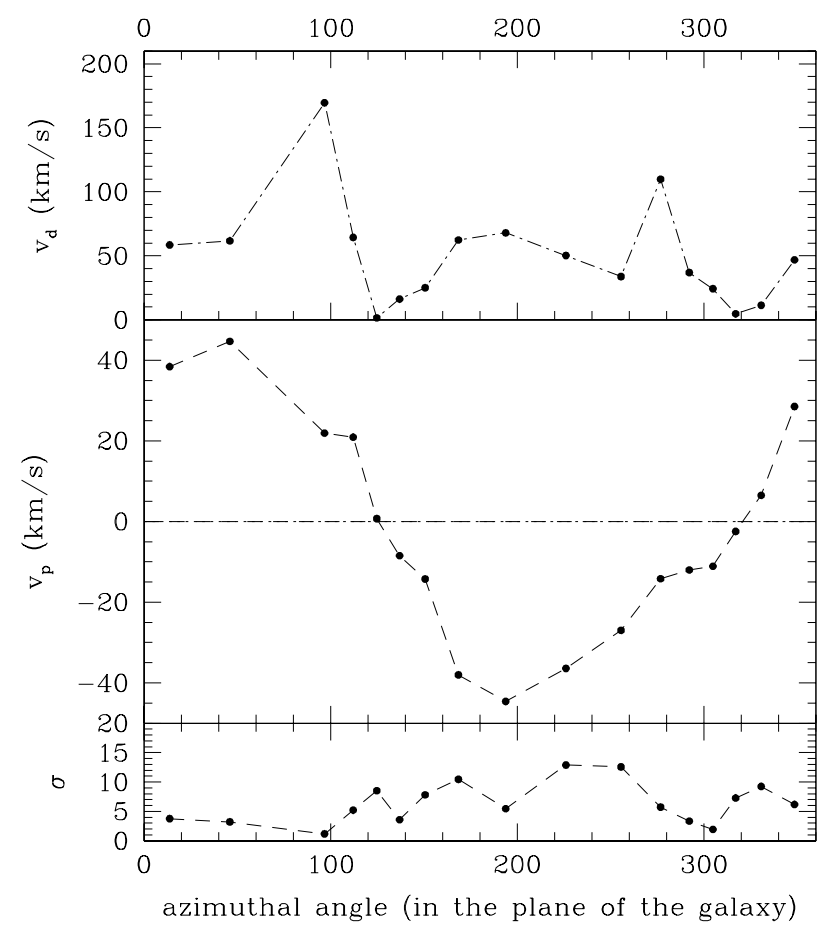

Fig. 17. Mean modulus of the velocity gas flow around the bar, as a function of azimuthal angle in the plane of NGC 3359 (upper panel); mean projected velocity for each sector of the projected bar annulus as a function of azimuthal angle (middle panel) and estimated errors (lower panel). The angles are measured in a clockwise direction in the plane of the galaxy

- The velocity dispersion map has been obtained and its radial distribution has been analyzed. We find a constant mean value with the radius of around $15 \mathrm{~km}$ $\mathrm{s}^{-1}$; the peaks in velocity dispersion coincide with the luminosity peaks, i.e. correspond to the brightest HII regions;

- We have accurately analyzed the non circular motions around the bar and a simple model has been developed, which is able to explain the velocity gradients in this region as gas flowing in elliptical orbits around the bar. This, together with the presence in the bar region of strong star formation fits the general scenario of a bar in the process of formation.

Acknowledgements. The William Herschel Telescope is operated on the island of La Palma by the Royal Greenwich Observatory in the Spanish Observatorio del Roque de los Muchachos of the Instituto de Astrofísica de Canarias. We thank F. Garzón for his help with the English version of this paper. This work was partially supported by the Spanish DGICYT (Dirección General de Investigación Científica y Técnica) via Grants PB91-0525, PB94-1107 and PB97-0219. This research has made use of the NASA/IPAC Extragalactic Database (NED) which is operated by the Jet Propulsion Laboratory, California Institute of Technology, under contract with the National Aeronautics and Space Administration.

\section{References}

Ball R., 1986, ApJ 307, 453

Ball R., 1992, ApJ 395, 418

Begeman K., 1989, A\&AS 223, 47

Combes F., Becquaert J.F., 1997, A\&A 326, 554

de Vaucouleurs G., de Vaucouleurs A., Corwin H.G., et al., 1991, Third Reference Catalogue of Bright Galaxies (RC3). Springer, New York

Friedli D., Benz W., 1993, A\&A 268, 65

Friedli D., Benz W., Kennicutt R., 1994, ApJ 430, L105

Gottesman S.T., 1982, AJ 87, 751

Roberts W.W. Jr., Huntley J.M., van Albada G.D., 1979, ApJ 233,67

Knapen J.H., 1997, MNRAS 286, 403

Knapen J.H., 1998, MNRAS 297, 255

Knapen J.H., Shlosman I., Heller C.H., 1999, MNRAS (in press)

Martin P., Roy J.R., 1995, ApJ 445, 161

Norman C.A., Ikeuchi S., 1988, Lecture Notes in Physics, Vol. 306. Springer-Verlag, Berlin, New York

O'Dell C.R., Townsley L.K., 1988, A\&A 198, 283

Osterbrock D.E., 1989, Astrophysics of Gaseous Nebulae and Active Galactic Nuclei. Mill Valley, University Science Books

Rots A.H., 1990, ApJ 100, 387

Rozas M., Sabalisck N., Beckman J.E., Knapen J.H., 1998a, A\&A 338, 15

Rozas M., Knapen J.H., Beckman J.E., 1998b, MNRAS 301, 631

Rozas M., Zurita A., Beckman J.E., 1999, A\&A (in press) (Paper I)

Rubin V.C., Burstein D., Ford W.K., Thonnard N., 1985, ApJ 289,81

Sempere M.J., Rozas M., 2000, A\&A (submitted)

Spitzer L., 1978, Physical Processes in the Interstellar Medium. Wiley, New York

van der Kruit P.C., Shostak G.S., 1982, A\&A 105, 351

Vila Costas M.B., Edmunds M.G., 1992, MNRAS 259, 121

Visser H.C.D., 1980, A\&A 88, 149

Zurita A., Rozas M., Beckman J.E., 2000 (in preparation) 